\title{
RESULTS FROM DETAILED STUDIES OF X-RAY SOURCES
}

\author{
R. GIACCONI \\ (American Science and Engineering, Cambridge, Mass., U.S.A.)
}

Since the results of early surveys revealed the existence of cosmic X-ray sources (Giacconi and Gursky, 1965), the major effort of X-ray astronomy has been devoted to the investigation of their nature. With the exception of the X-ray source in the Crab Nebula (Bowyer et al., 1964), an object well known prior to the discovery of its X-ray emission, the galactic X-ray sources which have been observed do not appear to coincide with conspicuous visible or radio objects.

The attempt to study their nature has proceeded along two main directions:

(a) Accurate determinations of celestial coordinates for the X-ray emitters permit the identification of their visible and radio counterparts. When a likely candidate is discovered, the techniques of optical astronomy yield information regarding the spectrum, size, distance, temporal variations and polarization of the radiation emitted by the object.

\section{Table 1}

\section{Position and intensity of observed $\mathrm{X}$-ray sources}

\begin{tabular}{|c|c|c|c|c|c|}
\hline & $l^{\mathrm{II}}$ & $\Delta l$ & $b^{\mathrm{II}}$ & $\begin{array}{l}\text { Intensity } \\
2-5 \mathrm{keV}\end{array}$ & $\begin{array}{c}\text { Other } \\
\text { Designation }\end{array}$ \\
\hline GX-14.1 & $-14 \cdot 1^{\circ}$ & $\pm \mathbf{0 . 2 5 ^ { \circ }}$ & $0+10^{\circ}$ & $0.11 \mathrm{cts} / \mathrm{cm}^{2}-\mathrm{sec}$ & \\
\hline GX-12.9 & -12.9 & \pm 0.3 & $>10$ & 0.33 & \\
\hline GX-10.7 & $-10 \cdot 1$ & 0.1 & $0+5$ & 0.90 & Lockheed-3 \\
\hline GX-5.6 & -5.6 & \pm 0.2 & $0 \pm 10$ & 0.11 & \\
\hline GX-2.5 & -2.5 & $\pm 0 \cdot 15$ & $0 \pm 10$ & $0 \cdot 40$ & \\
\hline Sco $X-1^{a}$ & -0.9 & - & $23 \cdot 8$ & $19+5$ & Sco XR-1 \\
\hline $\mathrm{GX}+2 \cdot 6$ & $2 \cdot 6$ & \pm 0.1 & $0+5$ & 0.68 & \\
\hline $\mathrm{GX}+5 \cdot 2$ & $5 \cdot 2$ & +0.1 & $-1+4$ & 1.24 & Lockheed-4 \\
\hline $\mathrm{GX}+9 \cdot 1$ & $9 \cdot 1$ & \pm 0.1 & $2 \cdot 54$ & 0.84 & Lockheed-5 \\
\hline $\mathrm{GX}+13.5$ & $13 \cdot 5$ & -0.1 & $-4+8$ & 0.37 & Lockheed-6 \\
\hline $\mathrm{GX}+16.7$ & $16 \cdot 7$ & \pm 0.1 & $4 \cdot 0+3$ & 1.25 & Lockheed-7 \\
\hline $\mathbf{G X}+36 \cdot 3$ & $36 \cdot 3$ & +0.2 & $9^{\circ} \pm 8$ & 0.15 & Ser XR-1 \\
\hline$G X+48 \cdot 7$ & $48 \cdot 7$ & +0.2 & $0 \pm 15$ & 0.19 & \\
\hline Cyg X-1 & 71.4 & $\mathrm{~b}$ & $3 \cdot 1$ & 0.40 & Cyg XR-1 \\
\hline Cyg X-2a & $87 \cdot 4$ & - & $-11 \cdot 3$ & 0.36 & Cyg XR-2 \\
\hline Cyg X-3 & $80 \cdot 0$ & b & 0.7 & 0.13 & \\
\hline Cyg X-4 & $82 \cdot 9$ & b & -6.4 & 0.05 & \\
\hline
\end{tabular}

Perek (ed.), Highlights of Astronomy, 192-201. () I.A.U. 
(b) The properties of the X-ray emitter are investigated by careful measurement of the angular size of the $\mathrm{X}$-ray emitting region, its detailed spectrum and its polarization and variability.

The most fruitful approach to date has been the one under (a). By means of accurate positional determinations we have been able to identify the optical counterparts of Sco X-1 and Cyg X-2 in addition, of course, to the Crab Nebula. It must, however, be realized that for the majority of the X-ray sources presently known, this approach
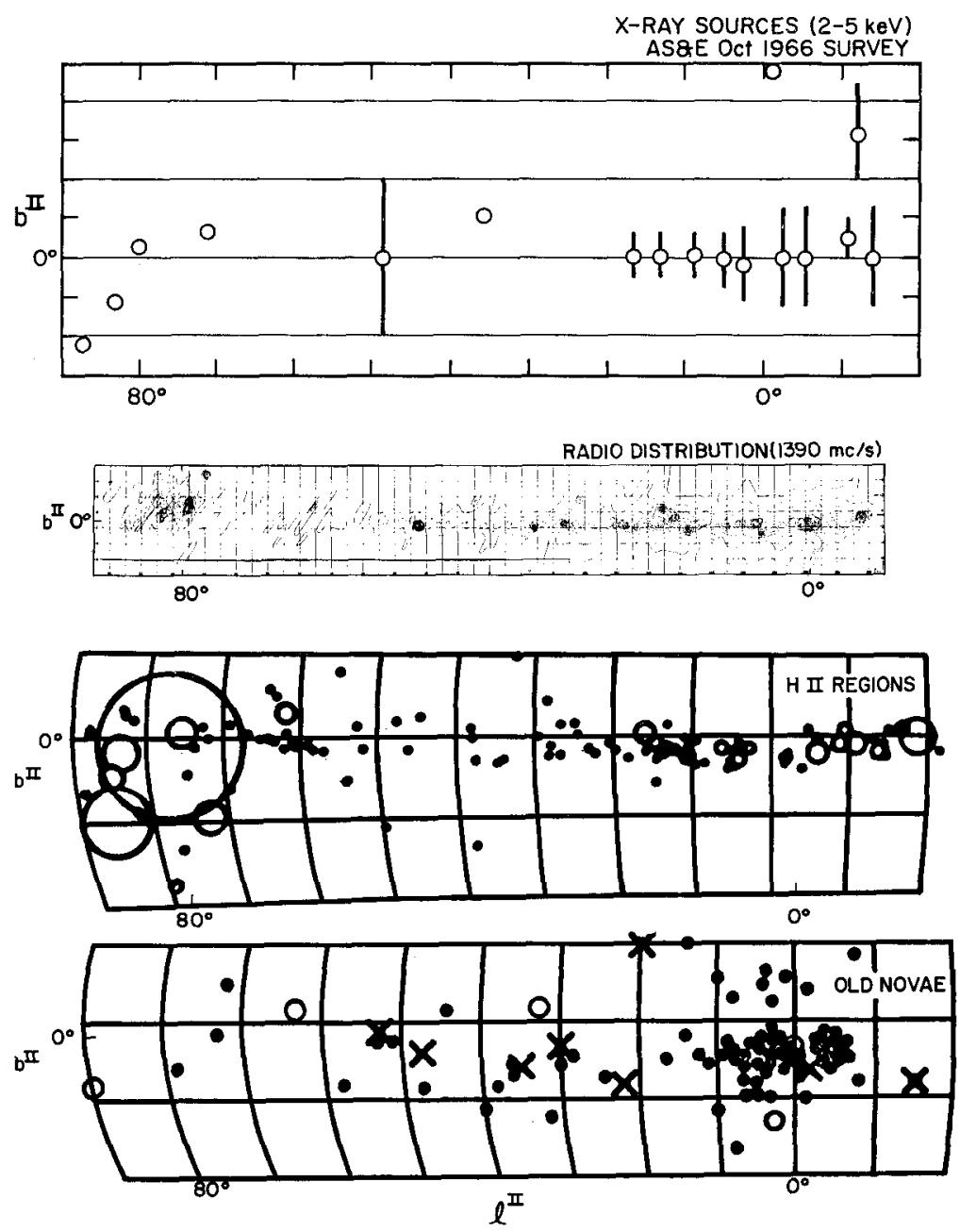

FIG. 1. Comparison of the distribution of X-ray sources with known galactic objects. The radio distribution is taken from Westerhout, the $H$ II distribution is that prepared by Plaut, and old-novae distribution is that of Sharpless. The angular scale is identical in all four plots. The X-ray positions shown are based on a survey of the galactic equator by the ASE group from a sounding rocket flown on October 11, 1966. 
may not be possible due to the relative low brightness of their optical counterparts and the fact that the sources appear to be located in regions of high obscuration.

For a number of the presently known sources positions have been accurately determined in at least one direction. Table 1 summarizes the results for sources whose position is known with accuracies of a few minutes of arc in at least one direction. Inspection of Table 1 shows that for only five of the sources listed, i.e. Sco X-1, Cyg X-1, Cyg X-2, Cyg X-3, and Cyg X-4, in addition to Crab, do we know positions in two dimensions with minutes of arc accuracy (Gursky et al., 1967). Only for these sources have we been able to search with confidence for optical counterparts.

The remainder of the data has been used to search for correlations with singular

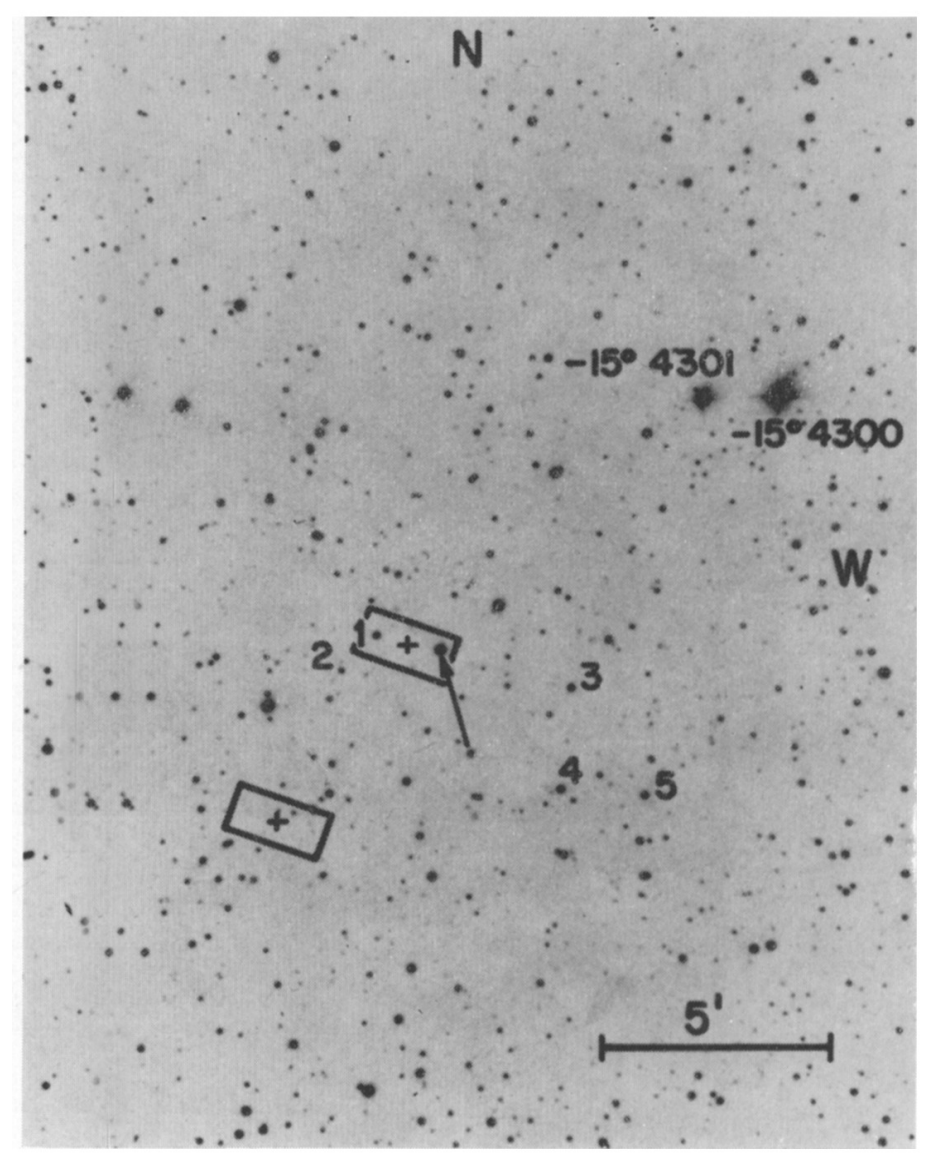

FIG. 2. Photograph of the region containing the $X$-ray position of Sco $X-1$ reproduced from a Palomar Sky survey print. The two equally probable X-ray positions as determined by Gursky et al. (1966) are marked by crosses surrounded by a rectangle of 1 by 2 min of arc. The object identified as the optical counterpart of the X-ray source is shown by the arrow. Other marked stars were used for comparison photometry. 
features of the galaxy. We find that the distribution of the sources in galactic longitude coincides with the distribution of $\mathrm{H} 1 \mathrm{l}$ regions and of $\mathrm{OB}$ associations. We conclude that most of the observed X-ray sources occur in the spiral arms of our galaxy. The remarkable correlation is shown in Figure 1.

For those sources for which positions are accurately known, a search for optical counterparts has been undertaken. This search has been successful in the case of

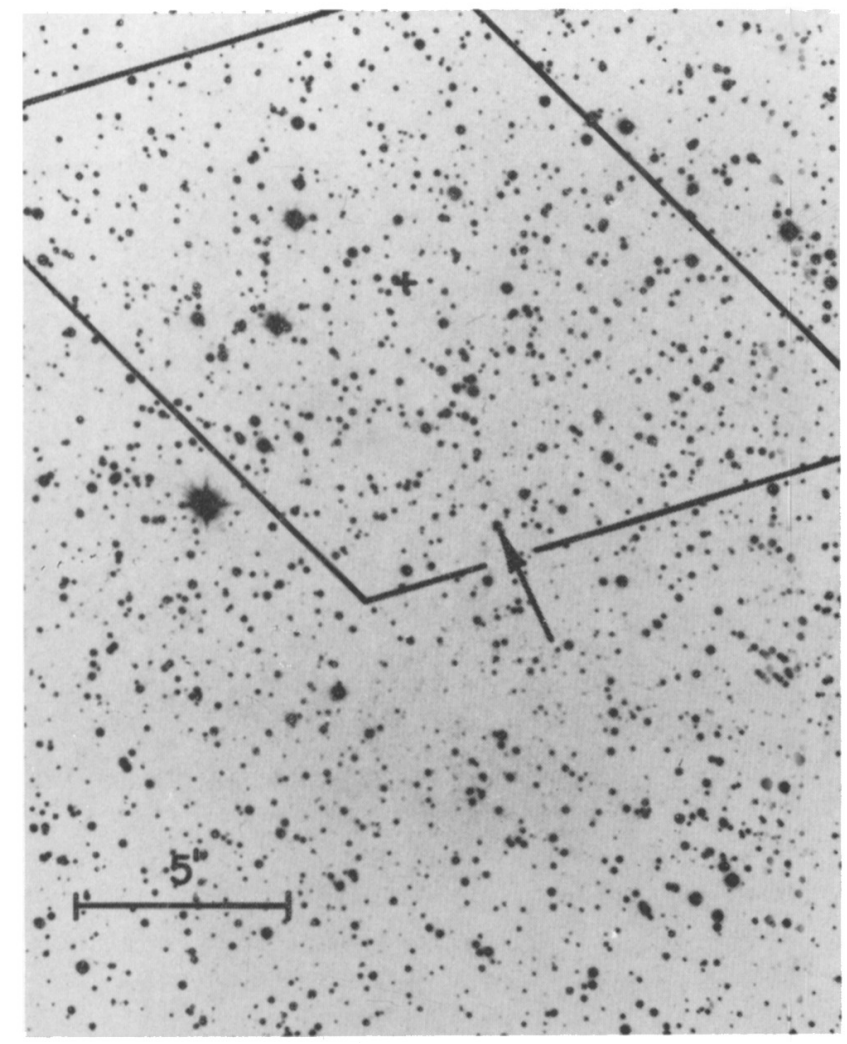

FIG. 3. The region containing the X-ray source Cyg X-2 taken from a Palomar Sky survey print. $X$-ray position is marked by the cross and the area of uncertainty is the trapezoid. The optical candidate is marked by an arrow. The elongation of the optical image of the candidate object is caused by a neighboring 19 th mag star and is not associated with the candidate object itself.

Sco X-1 (Gursky et al., 1966; Sandage et al., 1966) and Cyg X-2 (Giacconi et al., $1967 a, b)$. In Figures 2 and 3 the portion of sky containing these sources is shown together with the $\mathrm{X}$-ray location and the optical candidate. In Figure 4 the location of the X-ray source in Crab (Oda et al., 1967) is shown superimposed on a visible light photograph. For Cyg X-1, Cyg X-3 and Cyg X-4 no optical candidate has been 
identified. For Cyg X-1 and Cyg X-3 spectral measurements of their X-ray spectra have been carried out.

In brief, a summary of the results of the optical and X-ray measurements of the more intensively studied objects are as follows:

Sco $X-1, C y g X-2$ : Both objects exhibit a relatively soft X-ray spectrum. The spectral shape can be described by an exponential with characteristic temperatures of $5 \times 10^{7}$ for Sco X-1 and $36 \pm 6 \times 10^{7}$ for Cyg X-2. No absorption features have been detected in the X-ray spectra at the lowest energy detected $(\sim 1 \mathrm{keV})$. Both

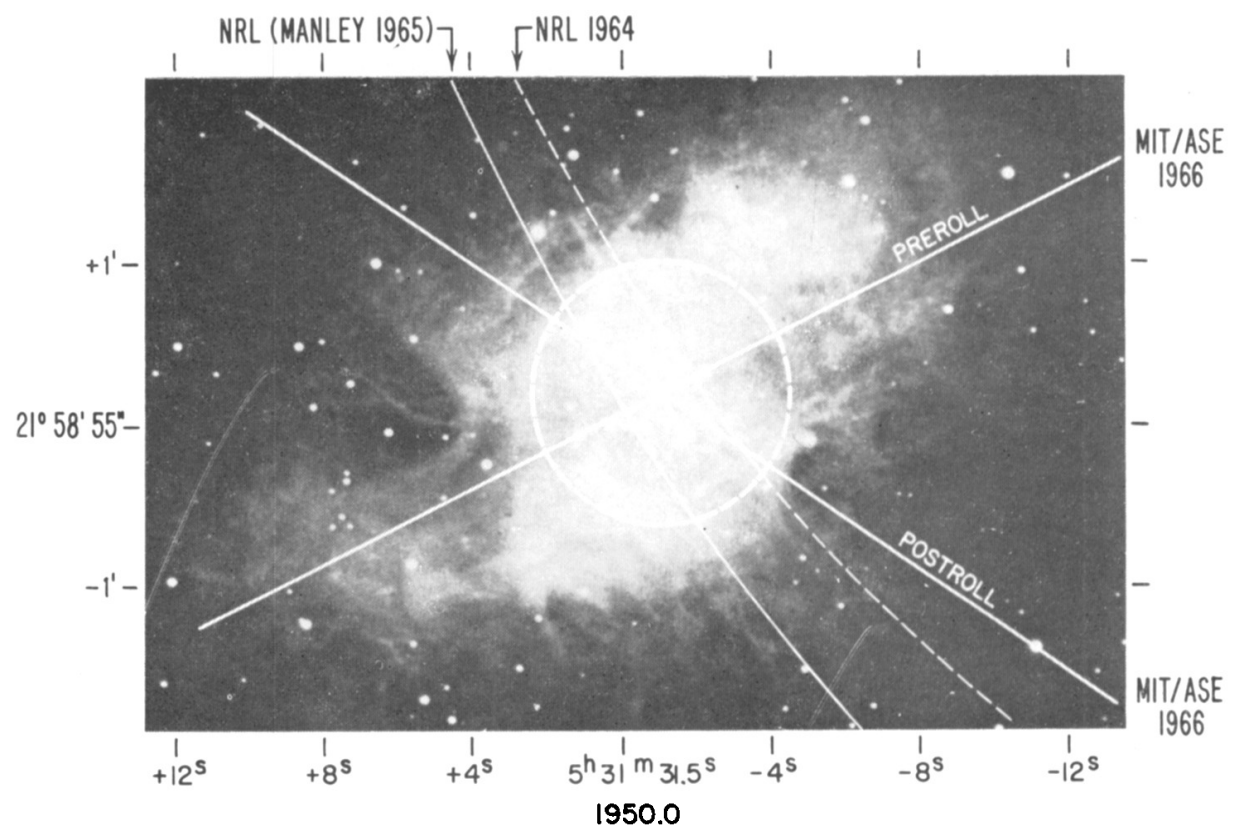

FIG. 4. Summary of results pertaining to the position of the X-ray source in the Crab Nebula. The dashed curved line is the line of position obtained from a lunar occultation experiment by Bowyer et al. (1964), and the solid curved line is the same result corrected by Manley and Ouellette for parallax due to the motion of the rocket. The intersection of the two MIT/AS\&E lines of position is at (J950.0) $\alpha=5^{h} 31^{m} 30^{s}, \delta=21^{\circ} 59.1^{\prime}$. The $100^{\prime \prime}$ diameter circle is an idealization of the source region. The origin of the coordinate system is the Southwest component of the central double star.

sources are at relatively high galactic latitude. The optical candidates appear as blue starlike objects whose magnitude agrees with the one predicted by extrapolation from $X$-ray to visible according to an exponential spectral law. It follows that the energy output in X-rays exceeds by a factor of 1000 the combined optical and radio outputs. The visible light spectrum (Sandage et al., 1966) from Sco X-1 exhibits characteristic emission lines of hydrogen and higher $Z$ elements in an excited state. Both the line emission and continua appear to vary rapidly with time. The spectrum of Cyg X-2 
shows HeII in emission (Lynds, 1967) and also appears to be variable. The distances of Sco X-1, estimated on the basis of the Ca K absorption feature, as well as on the absence of proper motion (Luyten, 1966), yield distances greater than about $\frac{1}{2} \mathrm{kpc}$. No generally accepted model of either of the two objects yet exists. It is, however, generally accepted that the X-ray emission occurs in a medium optically thin to its own radiation. In Figure 5 the measured X-ray spectrum of Sco X-1 is shown.

The two objects (Sco X-1 and Cyg X-2) have several common characteristics and may be representative of a class of celestial X-ray emitters which includes a large fraction of all known X-ray objects.

Crab Nebula: An X-ray spectrum of the Crab Nebula is shown in Figure 6. The

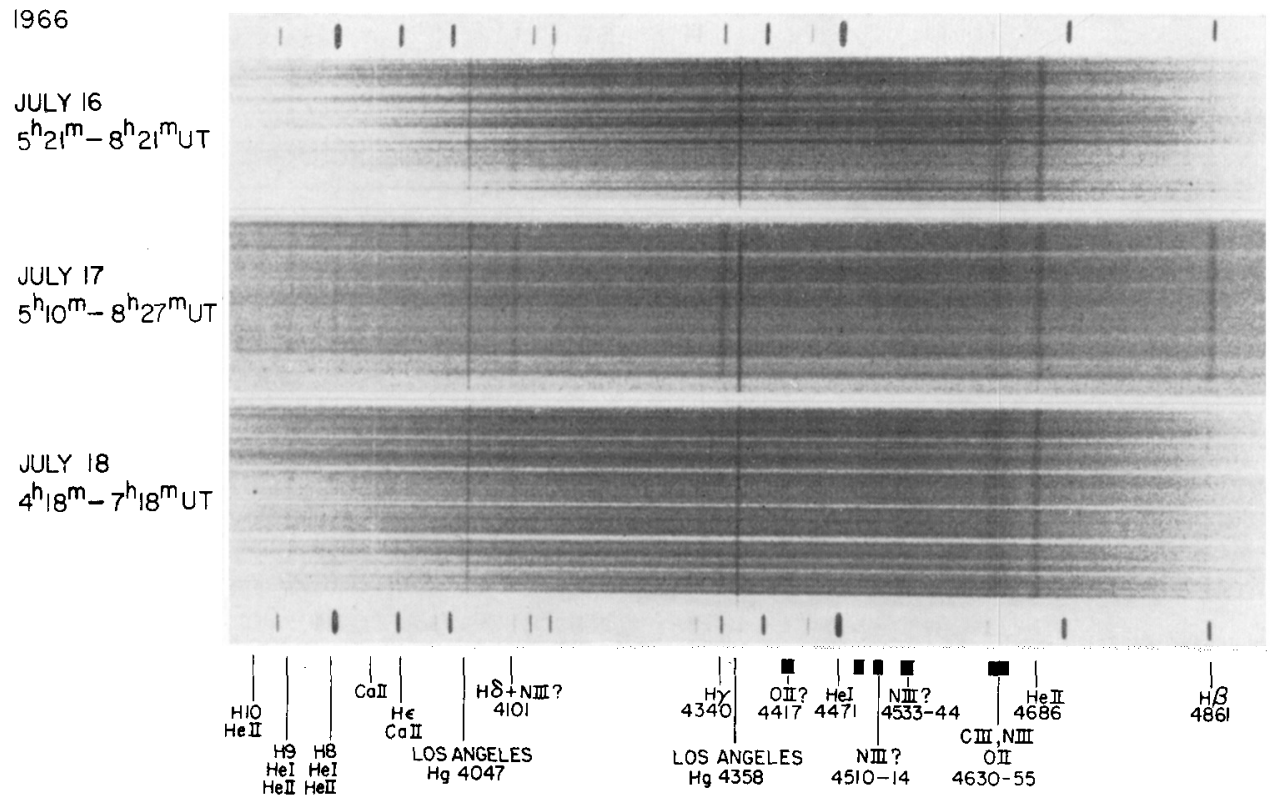

Fig. 5. Spectra of the Sco X-1 optical counterpart, obtained by A. Sandage at Mount Wilson and Palomar Observatories. From top to bottom, the exposures were on July 16, 1966, from 5:21 to 8:21 Universal time; July 17; 5:10 to 8:27; and July 18, 4:18 to 7:18.

fact that a smooth extrapolation from the radio and visible to the X-ray spectrum can be made (Woltjer, 1964) is believed to strongly favor a common synchrotron emission process by relativistic electrons. The spectrum can be described by a power law spectrum of the form $E^{-\alpha}$ where $\alpha$ has the value of $1 \cdot 1$. The X-ray source in Crab is extended ( $\sim 2$ min of arc). It has been accepted that Crab may be representative of X-ray emission from supernova remnants. It should be noted that the Kepler supernova has not been observed to be an X-ray emitter. Tentative evidence exists for X-ray emission from Cas A and Tycho's supernova (Friedman et al., 1967). 
Cyg $X-1$ : No visible object has yet been identified as the optical counterpart of Cyg X-1. This is believed to be due to the great degree of obscuration in that direction in the sky; however, Cyg X-1 could be an extended object and have low surface brightness. No measurement has been made of its angular size. Its X-ray spectrum can best be fitted by a power law spectral shape $\left(E^{-\alpha}\right)$ with an exponent of $\alpha=\cdot 7$. No absorption feature has been observed (Gorenstein et al., 1967). The X-ray spectrum of Cyg X-1 is shown in Figure 7. Cyg X-1 appears to differ significantly from

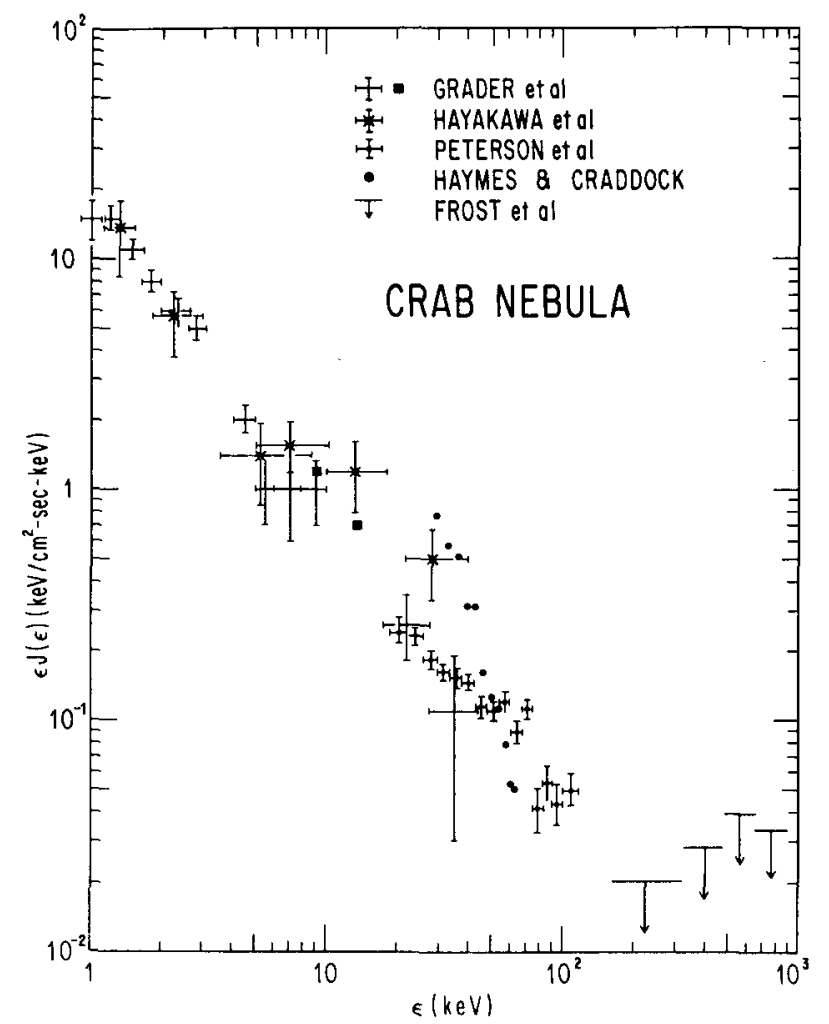

Fig. 6. A compilation by Gould of measurements of the spectrum of the Crab Nebula. The measurements of Grader and Hayakawa were obtained during sounding rocket flights. The others (Haymes, Peterson and Frost) were obtained during balloon flights.

Crab mainly in the absence of any observed radio emission. While the two sources have comparable $\mathrm{X}$-ray intensities, their radio emission differs by a factor of at least 500. This may indicate yet another type of X-ray emitter different from either supernova remnants or Sco X-1 like objects.

Cyg X-3: No visible counterpart of Cyg X-3 has yet been observed. This is also presumably due to obscuration since the object lies along one of the densest regions 
of the galaxy. Cyg X-3 is situated in the middle of the Cygnus- $\mathrm{X}$ radio region. However, the position is known accurately enough to exclude a coincidence with any of the known radio sources. The most interesting feature of the $\mathrm{X}$-ray emission from Cyg X-3 is that we have found evidence of absorption at energies of the order of $2.5 \mathrm{keV}$. This can be interpreted as absorption at the source or in the interstellar medium. The data (Clark et al., 1968) are insufficient to decide between a blackbody spectrum and an exponential spectrum with absorption features. The X-ray spectrum of Cyg X-3 is shown in Figure 8.

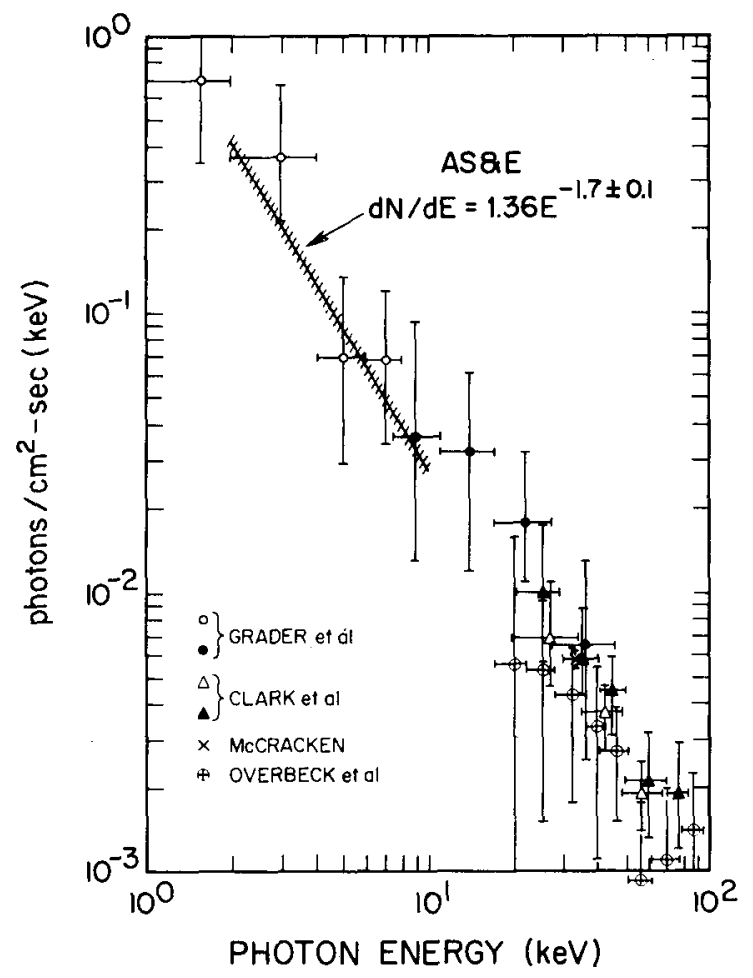

FIG. 7. A compilation of several rocket and balloon measurements on the spectrum of Cyg X-1 for a broad range of energies. The ASE result was obtained from the best fit of a power law spectrum to the data obtained in a rocket fight on October 11, 1966.

We find evidence of absorption also in the spectra of sources in the SagittariusScorpius region. The high degree of correlation between the observed X-ray absorption and the degree of optical obscuration for several sources strongly suggests that this absorption occurs in the interstellar space. Using accepted values of interstellar matter, density and cosmic abundance of elements we find that the degree of observed attenuation is consistent with the qualitative assignment of X-ray sources to the 
galactic spiral arms, although the data seem to require a greater X-ray absorptivity than conventionally computed (Gursky et al., 1967).

The several X-ray sources observed would then be situated at distances of several kiloparsec from us and would mainly occur in regions of high obscuration. Several different types of celestial X-ray emitters appear to exist and we are beginning to distinguish between Sco X-1, Crab and Cyg X-1-like objects.

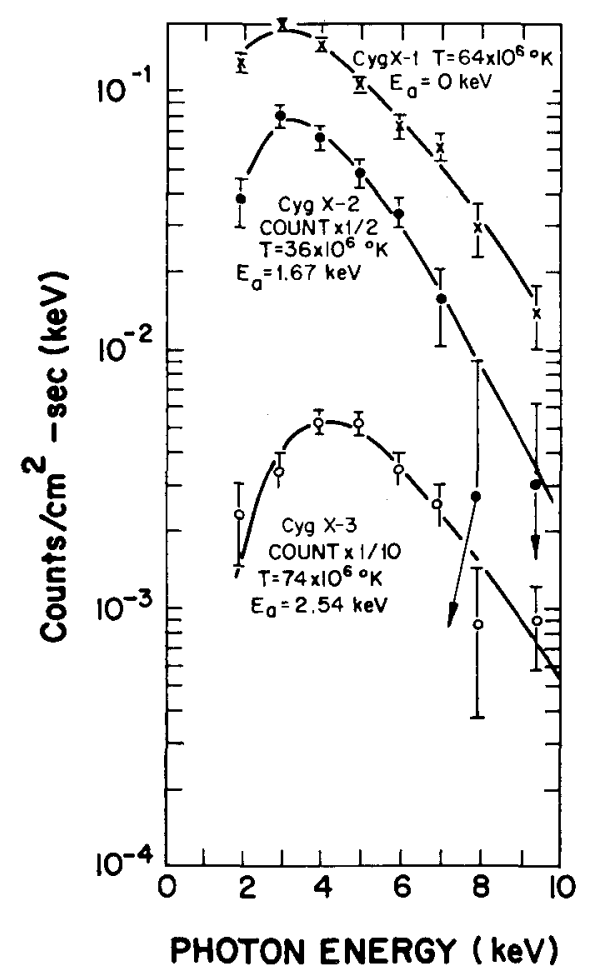

FIG. 8. The histograms of counting rate vs. energy are shown for three X-ray sources in Cygnus. The curves are continuous histograms which result from the calculated response of the detectors to an assumed photon distribution, $\mathrm{d} N / \mathrm{d} E \sim(E a / E)^{8 / 3} \exp (-E / K T) / E$. Various factors affecting the performance of proportional counters and the finite width of each energy bin are included. The quantities $E a$ and $T$ were left as free parameters. The values quoted are associated with the curve representing the best fit.

More refined measurements of the spectral characteristics, positions, angular size, structure, polarization and time variations of the X-ray emission from galactic objects are being carried out. The observation of characteristic X-ray lines in emission or of $\mathrm{X}$-ray absorption edges would contribute significantly to our understanding of the nature of X-ray objects. It is likely that real progress in X-ray astronomy in the future will largely depend on this and other refinements of the X-ray measurements themselves. 


\section{References}

Bowyer, S., Byram, E. T., Chubb, T. A., Friedman, H. (1964) Science, 146, 912.

Clark, G.W., Lewin, W.H.G., Smith, W.B. (1968) Astrophys. J., 151, 21.

Friedman, H., Byram, E.T., Chubb, T.A. (1967) Science, 156, 374.

Giacconi, R., Gursky, H. (1965) Space Sci. Rev., 4, 151.

Giacconi, R., Gorenstein, P., Gursky, H., Waters, J.R. (1967a) $\quad$ Astrophys. J., 148, L119.

Giacconi, R., Gorenstein, P., Gursky, H., Usher, P.D., Waters, J.R., Sandage, A., Osmer, P., Peach, J.V. (1967b) Astrophys. J., 148, L129.

Gorenstein, P., Giacconi, R., Gursky, H. (1967) Astrophys. J., 150, L85.

Gursky, H., Giacconi, R., Gorenstein, P., Waters, J.R., Oda, M., Bradt, H., Garmire, G., Sreekantan, B. V. (1966) Astrophys. J., 146, 310.

Gursky, H., Gorenstein, P., Giacconi, R. (1967) Astrophys., J. 150, L75.

Luyten, W.J. (1966) I.A.U. Astronomical Telegram, Circular \# 1980.

Lynds, C.R. (1967) 'Spectroscopic Observations of Cyg X-2'. Preprint.

Oda, M., Bradt, H., Garmire, G., Spada, G., Sreekantan, B.V., Gursky, H., Giacconi, R., Gorenstein, P., Waters, J.R. (1967) Astrophys. J., 147, 855.

Sandage, A., Osmer, P., Giacconi, R., Gorenstein, P., Gursky, H., Waters, J.R., Bradt, H., Garmire, G., Sreekantan, B. V., Oda, M., Osawa, K., Jugaku, J. (1966) $\quad$ Astrophys. J., 146, 316. Woltjer, L. (1964) Astrophys. J., 140, 1309. 